\title{
Der jüdische Salon in Berlin (und Wien) um 1800
}

\author{
Liliane Weissberg
}

\section{Theorie}

Die letzten Jahre des 18. Jahrhunderts waren für Friedrich Schleiermacher (1768-1834) eine erfolgreiche Zeit. Als protestantischer Prediger an der Berliner Charité erlangte er die Aufmerksamkeit der Berliner Gemeinde. Friedrich Schlegel lud ihn ein, in der 1798 mit seinem Bruder August Wilhelm gegründeten Zeitschrift Athenaeum zu veröffentlichen. 1799 erhielt Schleiermacher dann eine Einladung, den erkrankten Hofprediger Johann Peter Bamberger in Potsdam zu vertreten.

Sein Umzug von Berlin nach Potsdam tat seinen literarischen Arbeiten prinzipiell keinen Abbruch. Er beendete dort seine Reden über die Religion und arbeitete an einer Schrift zu einem Gesuch David Friedländers. Friedländer, der Vorsitzende der kleinen jüdischen Gemeinde zu Berlin, hatte sich 1799 mit einem Sendschreiben an den Berliner Oberkonsistorialrat Probst Wilhelm Teller gewandt und ihm vorgeschlagen, die Berliner Juden insgesamt zur Taufe zu bewegen; die Emanzipation der Juden sollte durch ihre Konversion ermöglicht werden. Der geplante religiöse Übertritt würde allerdings zu einem Christentum als Vernunftreligion im Sinne der Aufklärung geschehen; auf die besondere Rolle eines menschgewordenen Gottessohnes sollte dabei verzichtet werden. Probst Teller wandte sich gegen diesen Vorschlag, und auch Schleiermacher sprach sich dagegen aus.

Hinsichtlich einer Arbeit jedoch schien der Umzug nach Potsdam Schleiermacher nicht sonderlich zuträglich gewesen $\mathrm{zu}$ sein; er kam mit einer bestimmten Schrift nur langsam voran (Arndt 1997, 45-49). Es handelte sich dabei um seinen Versuch einer Theorie geselligen Betragens, bei dem Schleiermacher den Begriff der Geselligkeit philosophisch untersuchen wollte. Es ging dabei um nichts anderes als die Bestimmung des Menschen selbst. Ein Mensch, so führte er es auch in einem Brief an seine Schwester Charlotte aus, sollte in einem „Zustande moralischer Geselligkeit“ stehen (Schleiermacher, Brief v. 23.3.1799; KGA V/3, 49). Schleiermachers Aufsatz beginnt mit einem von Kant abgewandelten Imperativ: „Freie, durch keinen äußern Zweck gebundene und bestimmte Geselligkeit wird von allen gebildeten Menschen als eins ihrer ersten und edelsten Bedürfnisse laut gefordert" (Schleiermacher KGA V/3, 48). Ein Mensch sei in einer solchen Geselligkeit frei und gleichzeitig sei durch diese das „freie Spiel seiner Kräfte“ (Schleiermacher KGA V/3, 48) gefördert. Diese Geselligkeit wird durch Konversation geprägt, und das Gespräch sollte sich schwebend zwischen den durch die Gesellschaft festgelegten Polen bewegen. Eine solche Geselligkeit, wie sie Schleiermacher hier skizziert, ist vor allem bürgerlich. Sie setzt die Bildung des Einzelnen voraus und wendet sich gegen die Grenzen, die sich der Adel formal auferlegt. Eine höfische Gesellschaft kann nie 
„geräumig genug“ (Schleiermacher KGA V/3, 173) sein, um das freie Spiel zu erlauben.

Dabei ist Schleiermachers Begriff der Geselligkeit auch in einem anderen Sinne sozial bestimmt. Die Geselligkeit erscheint bei ihm als eine Art Kunst, in der gerade Frauen eine besondere Rolle spielen können, und dies ist in ihrer besonderen Situation begründet:

\begin{abstract}
Ich kann mich hiebei der Bemerkung nicht enthalten, ob nicht, wenn es anders wahr ist, daß die bessere Geselligkeit sich bei uns zuerst unter den Augen und auf Betrieb der Frauen bildet, dieses, wie so vieles andere Vortrefliche, in den menschlichen Dingen ein Werk der Noth ist? Die Frauen nämlich sind, wenn sie der hier getadelten Maxime folgen, weit übler daran, als die Männer, und ihre auf diese Art organisirten Gesellschaften müssen nothwendig die schlechtesten und gemeinsten seyn. Denn wenn der Mann auch von seinem Beruf spricht, so fühlt er sich doch von einer Seite noch frei, nämlich von der häuslichen; dagegen die Frauen, bei denen beides zusammenfällt, bei einer solchen Unterhaltung ihre Fesseln fühlen. Dies treibt sie dann weg unter die Männer, bei denen sie denn, weil sie mit dem bürgerlichen Leben nichts zu thun haben, und die Verhältnisse der Staaten sie nicht interessiren, jener Maxime nicht mehr folgen können, und eben dadurch, daß sie mit ihnen keinen Stand gemein haben, als den der gebildeten Menschen, die Stifter der besseren Gesellschaft werden. (Schleiermacher KGA V/3, 173)
\end{abstract}

Eine solche Geselligkeit war in Potsdam schlichtweg nicht vorhanden. Potsdam war ein Ort, an dem Friedrich II. seine Soldaten stationierte und einige Adelige um sich versammelte; Schleiermacher musste sich nach getaner Arbeit lediglich mit dem Kartenspiel im Hause Bamberger zufrieden geben (Arndt 1997, 47). Sein romantisches Modell einer Geselligkeit als Lebenskunst hatte dagegen ein Berliner Vorbild, die Treffen im Hause seiner Freundin Henriette Herz (1764-1847). Diese war natürlich nicht nur durch die „Fesseln“ ihres Geschlechts behindert, sondern ebenso durch jene ihrer Religion und Herkunft. Herz war die Tochter des jüdischen Arztes Benjamin de Lemos und seiner Frau Esther de Charleville und mit dem Arzt und Philosophen Marcus Herz (1747-1803) verheiratet, einem Schüler Kants, der als Nachfolger von de Lemos die Leitung des Jüdischen Krankenhauses in Berlin übernahm. Nicht nur als Frau und Nicht-Adelige, sondern auch als nicht emanzipierte Jüdin litt Herz unter den Restriktionen der Gesellschaft. Heute gilt Herz als die erste jüdische Salonnière Berlins, der weitere bald folgen sollten. Prädestinierte ihre doppelte Außenseitersituation - als Frau, als Jüdin - Herz dazu, Gastgeberin von Geselligkeiten im Schleiermacherschen Sinne zu werden?

\title{
Praxis
}

Für Schleiermacher gehörte die Geselligkeit zu den notwendigen Bedingungen eines bürgerlichen Individuums. Der Ursprung des Konzepts des ,Salons“ ist allerdings weder in Berlin noch im Bürgertum noch in den jüdischen Häusern zu suchen, deren Bewohner sozial und politisch nicht zum Bürgertum zählen konnten. 
Das Wort ,Salon“ ist der französischen Sprache entnommen. Dort erschien es zunächst 1664 und bezeichnete den Empfangsraum eines Schlosses; 1737 wurde ein Raum des königlichen Louvre so genannt, in dem Kunstausstellungen abgehalten wurden. Denis Diderot, der diese Kunstausstellungen beschrieb, nannte sie ebenfalls „Salons“, und das Wort wurde damit auch zu einem literarischen Begriff (Wilhelmy 2000, 16-32; Weissberg 2003). Der Begriff ,Salon` wurde gleichzeitig von französischen Adeligen des 17. und 18. Jahrhunderts übernommen. Da sie von der offiziellen Gesprächsrunde am Hofe meist ausgeschlossen wurden, bildeten sie ihre eigene, inoffizielle Gesprächsrunde in einem Wohnzimmer, das nun auch ,Salon` hieß. Unter dem Vorsitz einer Frau trafen sich Frauen zu einer Konversation über Kunst, Literatur, Geschichte und sogar Politik, die durch eine weiterführende und oft intensive Korrespondenz bereichert wurde. Der festumrissene, konkrete Raum (das Zimmer) wurde durch den imaginären Raum weiterer Begegnungen (den Brief) ergänzt. Durch Gespräche und Briefe konnten Beziehungen geknüpft werden; ein Netzwerk von Frauen entstand, die ihre eigenen intellektuellen Interessen - Politik, Kunst, Literatur, Musik - pflegen konnten. Der Raum des Salons wurde daher auch $\mathrm{zu}$ einem Bereich des Austauschs, der Unterhaltung wie Bildung ermöglichte und bestätigte. Und er war weiblich konnotiert.

Was sich in Berlin um 1800 entwickelte, war allerdings eine andere Art der Gegenkultur. Berlin stand dabei unter französischem Einfluss. Es lebten viele Hugenotten dort. Am preußischen Hof und in Adelskreisen wurde französisch gesprochen. Paris war darüber hinaus die kulturelle Hauptstadt dieser Zeit. Aber während der Pariser Salon von sozial privilegierten Frauen getragen wurde, die sich zwar gegen die Hofkultur wandten, aber selbst von Adel waren, luden in Berlin im späten 18. Jahrhundert vor allem jene Frauen zum Gespräch ein, die nicht zum Adel gehörten. Wilhelmy zeigt, dass sich diese Konstellation im späteren 19. Jahrhundert und mit der Zeit der Restauration ändern sollte, da diese Gesellschaften bald anerkannt wurden und eine Repräsentationsfunktion erhielten (Wilhelmy 1989). Um 1800 jedoch waren viele Gastgeberinnen noch nicht einmal Frauen des Bürgertums; die vielleicht bekanntesten Salons dieser Zeit wurden von Jüdinnen geleitet.

Damit änderte sich auch die Struktur des Salons. Im französischen Salon war eine gewisse Gleichberechtigung über den Raum des Salons hinaus gegeben; alle Frauen waren sozial höher gestellt. Eine Frau, die Gast in einem Salon war, konnte wiederum Gastgeberin spielen und andere Frauen einladen. Im Berliner Salon war diese Reziprozität nicht gegeben. Er öffnete sich zwar Menschen verschiedener Klassen und Berufe sowie verschiedener Herkunft und Religion, bestand aber auf keinem Modell, das gegenseitige Einladungen ermöglichte. Weiterhin war der jüdische Salon nur durch die Gastgeberinnen weiblich konnotiert, unter den Gästen selbst waren zahlreiche Männer. Hinsichtlich der jüdischen Gäste war dieser Salon allerdings geschlechtsbedingt. Er wurde vor allem von jüdischen Frauen besucht, die sich intensiver als ihre Väter, Ehemänner oder Brüder einer säkularen Bildung wid- 
men konnten (Richarz 1992); vor allem aber auch wohlhabendere jüdische Frauen, welche den Luxus besaßen, über ihre Zeit zu verfügen. Ihre männlichen Ehepartner und Verwandten waren vor allem Bankiers, Fabrikbesitzer und Händler, die den Salon kaum besuchen konnten oder wollten. Nichtjüdische Vertreter und Vertreterinnen der bürgerlichen Klasse oder des Adels konnten zwar ein jüdisches Haus betreten, aber selbst eine bekannte jüdische Gastgeberin war in einem gesellschaftlich etablierten Haus nur ungern gesehen. Die Salons waren Orte besonderer Begegnungen. So konnte ein Prinz seine Geliebte in einem dieser Salons treffen, sich allerdings an keinem öffentlichen Ort mit ihr zeigen.

Die Sprache des Salons unterschied sich von der des Hofes; es wurde vor allem Deutsch gesprochen. Nicht der Kaffee der Kaffeehäuser wurde gereicht, nicht die Schokolade des Hofes, sondern Tee. Das Gespräch, die Musik und die öffentliche Lektüre von Briefen oder Manuskripten stand im Vordergrund, nicht das Gastmahl. Für Hannah Arendt zeigte sich dieser Salon, wohl auch Schleiermachers Bestimmung folgend, als ein utopisch anmutender Freiraum, der eine deutsch-jüdische Symbiose vorspielen konnte (Arendt 1955 und 1959). Jede dieser Geselligkeiten musste aber auch die Realität außerhalb des Salon-Raumes deutlich machen. Wie Hannah Lotte Lund (Lund 2012) zeigt, gab es dabei allerdings auch konstante Bemühungen, den Raum des Berliner Salons jenseits des Zimmers und Hauses, ja sogar jenseits Berlins zu erweitern: Treffen fanden in Sommerhäusern der Gastgeber in Charlottenburg und auf dem Lande statt, in Badeorten wie Teplitz und Karlsbad, auf den Wegen von und zum Theater.

Henriette Herz, die ein Modell prägte, das inzwischen als ,jüdischer Salon` in die Kulturgeschichte einging, hatte für ihre Gesellschaften zwei weitere Vorbilder in Berlin. Das eine waren die Einladungen zu dem Philosophen Moses Mendelssohn (1729-1886), der trotz seiner beschränkten finanziellen Mittel sein Haus gerne Philosophen, Wissenschaftlern, Studenten öffnete; seine Ehefrau Fromet servierte dabei, sorgfältig abgezählt, Rosinen und Mandeln zum Gespräch und hielt sich sonst zurück. Das andere Vorbild war ihr Ehemann Marcus Herz, der im gemeinsamen Haus Vorlesungen zur Kantschen Philosophie oder zur Physik hielt. Der Berliner Bildhauer Johann Gottfried Schadow (1764-1850), der selbst eine aus einer Wiener jüdischen Familie stammende Frau, Mariane Devidels, im Herzschen Salon kennenlernen und dann heiraten sollte, gab den folgenden Bericht:

Marcus Herz als praktischer Arzt und als Verfasser der Abhandlung vom Schwindel, außerdem witziger Kopf, stand allgemein und auch bei seinen Kollegen in hoher Achtung. Des Lavoisier Entdeckungen hatten ihn dermaßen bezaubert, daß er kostbare Apparate herbeischaffte, um uns an Nachmittagen Experimente vorzutragen. Als leidenschaftlicher Tabakraucher blieb er Herr in seinem Zimmer. Hier empfing er an den Gesellschaftsabenden junge Ärzte, durchreisende Gelehrte, unter denen nur namentlich anzuführenden Geheimrat Selle, der Königliche Leibarzt; N. Kunth, Erzieher der berühmten Brüder Wilhelm und Alexander von Humboldt; Klaproth; Staatsrat Karsten etc. Im Salon der Hausfrau daneben waren zugleich mehrere jüngere Männer, der deutschen Dichtkunst ergeben, das hierin Neuerscheinende beibringend, be- 
sprechend, rezitierend und kritisierend. Von den vielen Namen sind wenige in der Erinnerung geblieben: der Schwede Brinckmann, von dem Verse im Druck erschienen; Woltmann, auch Geschichtsschreiber; einer names Meyer, Almanach versifax; Meyering; von Kleist und die Gebrüder Grafen Dohna; der ältere Graf wurde Staats-Minister, der jüngere bekleidete mehre Gesandtschaften. Der berühmte Schleiermacher kam mehre Jahre später erst hinzu. (Schadow 1849, XIX-XX)

Die strikte räumliche Trennung dieser Parallelwelten mag Fiktion sein, nicht aber der unterschiedliche Charakter der Zusammenkünfte. Obwohl Mendelssohn wie Marcus Herz Einladungen in ein jüdisches Heim aussprachen, waren ihre Treffen oder Veranstaltungen keine Geselligkeiten im Schleiermacherschen Sinn. Sie waren der aufklärerischen Bildung verbunden und nicht dem geforderten wechselseitigen, freischwebenden Gespräch. Bei einer Einladung zu Henriette Herz sollte sich dies nun ändern. Bildung wurde zum Austausch statt einseitiger Belehrung; Gesprächsthemen wurden selten vorgegeben und konnten rasch wechseln. Die Art der Formulierung, der besondere Witz einer Darstellung konkurrierten mit dem Inhalt.

Wenn Schleiermacher daher in Potsdam die Geselligkeit vermisste, so war dies eine Geselligkeit, die wenig mit der adeligen Welt Preußens gemein hatte. Der Berliner Salon hatte dabei verschiedene Funktionen. Er gab Gelegenheit zu einer Konversation, die nach Schleiermacher das bürgerliche Individuum bestimmte und gleichzeitig verwirklichen sollte. Er wurde ein Ort der Begegnung, der klassenübergreifend war und sich jenseits üblicher Moralvorstellungen stellte. Es konkurrierten dabei auch zwei neue Wirtschaftsordnungen. Für wohlhabende jüdische Frauen wurde der Salon nicht nur zu einer Gelegenheit erträumter Emanzipation, sondern bisweilen auch zu einer Chance, sie auf eine besondere Weise zu verwirklichen. Wie Deborah Hertz (Hertz 1988/1991) in ihrer Studie ausführlich belegen konnte, konvertierten viele dieser Frauen zum Christentum und heirateten einen christlichen Mann, den sie in diesen Salons kennenlernt hatten. Sie heirateten bisweilen in den Adel ein. Sara Meyer (1763-1828) wurde Baronin von Grotthus, ihre Schwester Mariane (1770-1812), die den Fürsten Heinrich von Reuss heiratete, eine Prinzessin von Eybenberg. Verarmte Adelige konnten wiederum nicht nur einen Titel bieten, sondern mit dem Vermögen dieser Frauen auch ihre eigenen Güter konsolidieren. Das neue Phänomen des Salons bestätigte damit keine erträumte romantische Geselligkeit und die Macht des aufsteigenden Bürgertums, sondern eine Gesellschaftsordnung alter Art. Die Sehnsucht jüdischer Salonnières galt dem Adel.

Herz, die auch nicht sehr wohlhabend war und nach dem Tod ihres Mannes von einer Witwenrente leben mußte, folgte diesem Weg nicht. Zwar sollte sie letztendlich zum Christentum konvertieren, aber einen Heiratsantrag des Grafen Alexander von Dohna wies sie ab. Brendel (später Dorothea) Veit (1764-1839), die älteste Tochter Mendelssohns, lernte im Hause Herz Friedrich Schlegel kennen. Sie verließ ihren Ehemann, den Bankier Simon Veit, und lebte fortan mit Schlegel in Berlin, Jena und Paris zusammen, wo Dorothea zum Protestantismus konvertieren und Schlegel 
heiraten sollte. Beide konvertierten später in Köln zum Katholizismus. Sara (Zerelche) Itzig (1761-1854), eine Tochter des Hofjuden Daniel Itzig, der für seine Dienste für den preußischen König schon früh als erster und einziger Berliner Jude ein Emanzipationspatent erhielt, blieb Jüdin. Sie war mit dem Bankier Samuel Salomon Levy verheiratet. Itzig lud zu Gesellschaften ein, die sich oft durch musikalische Darbietungen auszeichneten. Ihr Neffe Felix Mendelssohn-Bartholdy, ein Enkel Moses Mendelssohns, teilte Itzigs Interesse an der Musik von Johann Sebastian Bach und der Bachschen Familie. Itzig förderte seinen musikalischen Werdegang. Mendelssohn-Bartholdys Mutter Lea (Lilla) Salomon, verheiratet mit Moses Mendelssohns Sohn Abraham, der ebenfalls ein Bankier war, lud zu musikalischen Darbietungen ein wie zu Gesprächen über Musik. Friederike (Fradchen) Marcuse (später Friedrika Liman) war ein häufiger Gast im Hause Levin und anderen Gesellschaften. Sie ließ sich von ihrem Ehemann Abraham Nathan Liepmann scheiden und lebte zunächst mit der Schauspielerin Friderike Unzelmann, dann mit der Sängerin Anna Milder zusammen.

Wie den französischen Salon, so begleitete auch den Berliner Salon eine rege Briefkultur. Dies wird besonders im Fall der wohl bekanntesten Berliner Gastgeberin deutlich, Rahel Levin (1771-1833), später verheiratet mit Karl August Varnhagen von Ense. Tausende ihrer Briefe sind bis heute erhalten. Sie wurden bereits zu ihren Lebzeiten von Varnhagen gesammelt und nach ihrem Tod in ersten Sammlungen vorgelegt.

Levin festigte ihren Ruf als bedeutende Gastgeberin und Korrespondentin bereits in jungen Jahren. Kurz nach dem Tod ihres Vaters lud sie Gäste in das elterliche Haus, wahrscheinlich zunächst auch jene Schauspieler und Bürger, denen der Vater als Bankier zuvor behilflich war. Im Unterschied zu Herz, die durch ihre außerordentliche Schönheit sowie große Sprachkenntnisse glänzen konnte, und Sara Itzig, deren Musikalität bekannt war, stach Levin durch ihren Witz und ihre Schlagfertigkeit hervor; durch eine Originalität des Ausdrucks, der sich in den erhaltenen Briefen widerspiegelt. Wie bei anderen Gastgeberinnen gab es auch bei Levin einen ,jour fixe‘, an dem man sich traf. Und obwohl Rahel oft von ihren „Dachstubenwahrheiten“ schrieb, war der Hinweis auf ihr eigenes Zimmer eher ein Bescheidenheitstopos. Ihre Geselligkeiten fanden wie sonst auch üblich im repräsentativeren Wohnzimmer des Hauses statt, und ihre Familie nahm oft an ihnen teil. Levins Salon konnte wohl die prominentesten Gästen aufweisen. Obwohl viele Personen (etwa die Gebrüder Alexander und Wilhelm von Humboldt, der schwedische Diplomat Gustav von Brinkmann) mehrere der Salons frequentierten, war in Levins Salon ein preußischer Prinz, Louis Ferdinand, gelegentlich zu Gast; Rahel war mit seiner Geliebten Pauline Wiesel eng befreundet. Auch dies gab ihrem Salon einen besonderen Status.

Die soziale Freizügigkeit dieser Gesellschaften wurde 1806 beendet, als die napoleonische Armee in Berlin einmarschierte. Die folgenden Jahre wurden von einem preußischen Patriotismus bestimmt und von den Restaurationsbestrebungen nach den Kriegen gegen Frankreich. Gesellschaften in jüdischen Häusern waren nun 
nicht mehr attraktiv, und es wurde stärker auf Standesunterschiede hingewiesen. Herz zog sich bereits 1803, nach dem Tod ihres Mannes, als Gastgeberin zurück; Rahel beendete um 1806 ihre Geselligkeiten. Zwar öffnete sie ihr Haus 1814, nach ihrer Heirat mit Varnhagen, erneut, aber die politische Situation machte sich auch hier bemerkbar - dies obwohl preußische Juden 1812 emanzipiert wurden und Rahel selbst kurz vor ihrer Eheschließung konvertieren sollte.

Wilhelmy (1989) sammelt viele Namen der jüdischen (und nichtjüdischen) Gastgeberinnen und Gäste der Berliner Salons des späten 18. und 19. Jahrhunderts. Unter den Gästen und Gastgeberinnen wäre noch Rebecca Salomon, später Saaling (1783-1850) hervorzuheben, die sich nach ihrer Scheidung von Moses Friedländer, einem Sohn David Friedländers, den Namen Regina Frohberg gab. Unter diesem Namen veröffentlichte sie Unterhaltungsromane; Schmerz der Liebe (1810) gilt als ein Schlüsselroman des Levinschen Salons (Weissberg 2001). 1813 zog sie nach Wien und besuchte die dortigen Gesellschaften.

In Wien hatte sich inzwischen ein eigener jüdischer Salon etabliert. Daniel Itzig verheiratete zwei seiner Töchter nach Wien: Sara Itzigs Schwester Fanny (Vögele) (1757-1818) heiratete 1776 den Wiener Kaufmann Nathan Arnstein; Cäcilie (Zippora) Itzig (1760-1836) 1800 den Kaufmann Bernhard Eskeles. Sowohl Arnstein wie auch Eskeles konnten vom österreichischen Kaiser ein Adelspatent erwirken; sie erhielten den Titel eines Barons, ohne dass sie zum Christentum konvertieren mussten. Fanny von Arnstein wie auch Cäcilie von Eskeles wurden in Wien für ihre Gesellschaften bekannt. Durch Reisen und Korrespondenz hielten sie mit ihren Freundinnen, ihrer Familie und Berliner Salonnières Kontakt. Auch ihre Häuser öffneten sich Künstlern, Gelehrten und Adeligen, aber bedingt durch ihren gesellschaftlichen Status zeigten sich die Gastgeberinnen eher als Mäzeninnen im traditionelleren Sinne. Beide Frauen förderten zum Beispiel Ludwig van Beethoven, der 1792 von Bonn nach Wien gezogen war. Auch ihre Häuser sollten repräsentieren; beide bewohnten als Stadtpaläste bekannte Häuser. Das heutige Jüdische Museum Wien befindet sich im ehemaligen Palais Eskeles.

\section{Nachleben}

In den späten 1980er Jahren begann die intensive literatur- und kulturwissenschaftliche Aufarbeitung des Phänomens des jüdischen Salons in Berlin und auch Wien als Folge der Etablierung der Jüdischen Studien als Disziplin, als Erweiterung der Literaturwissenschaften auf Fragen der Sozialforschung und im Zuge der Wiederentdeckung der Schriften von Gastgeberinnen und Gästen, allen voran der Briefe Rahel Levins, die im Archiv der Berliner Staatsbibilothek aufbewahrt wurden und nach dem Krieg als verschollen galten. Sie wurden 1977 im Archiv der Bibliothek der Jagiellonen-Universität in Krakau wiederentdeckt. Barbara Hahn leitet seit den 1980er Jahren ein Editionsprojekt, das Teile der Korrespondenz zugänglich machte 
und Levins Ruf als Literatin festigte (vgl. Hahn 1990). Inzwischen gibt es vor allem zu Levin eine reiche Sekundärliteratur, welche ebenfalls die Forschung über den jüdischen Salon in Berlin beflügelte.

Die Soziologin und Historikern Deborah Hertz legte 1988 eine Studie vor, die auf den in den Kirchen geführten, erhaltenen Konversionsdaten um 1800 beruht und einen ersten Einblick in die soziale Zusammensetzung der Berliner Salons gibt und vor allem auch das Heiratsverhalten ihrer Mitglieder zu rekonstruieren sucht (Hertz 1988). Peter Seibert folgte kurz darauf mit einer Studie, die den literarischen Salon, und darunter vor allem den jüdischen Salon um 1800, als Forschungsthema etablieren sollte (Seibert 1993, 102-203). Wilhelmys monumentale Studie zum Berliner Salon (Wilhelmy 1989) folgte mit Einzelbiographien jüdischer wie nichtjüdischer Gäste und Gastgeberinnern, die vom späten 18. bis zum frühen 20. Jahrhundert das soziale Leben Berlins prägten. Eine revidiertere kürzere Fassung dieses Werkes (Wilhelmy-Dollinger 2000) legt einen weiteren Schwerpunkt auf die geografischen Zentren dieser Geselligkeiten.

In den letzten Jahrzehnten setzte jedoch vor allem eine Kritik an dem Begriff des ,Salons“ ein. Hahn (Hahn 2002, 75-98) beschreibt ihn als Mythos. Richtig ist, dass weder Gastgeberinnen noch Gäste die Gesellschaften in Berlin um 1800 Salons nannten; sie beschrieben sie lediglich als Teegesellschaften oder Teetische. Auch in Varnhagens Memoiren (Varnhagen 1987) erscheint dieser Begriff nicht. Dagegen tritt der Begriff im späten 19. Jahrhundert auf, als nach der Gründung des Deutschen Kaiserreiches und im Kontext antisemitischer Ausschreitungen die Gesellschaften jüdischer Gastgeberinnen zum ersten Mal wiederentdeckt wurden; sie wurden als Orte deutsch-jüdischer Symbiose nostalgisch verklärt (Weissberg 2003). Ein ähnliches Verlangen nach der Erfahrung eines glücklichen Zusammenlebens von Juden und Nichtjuden war auch in vielen Studien nach dem Zweiten Weltkrieg zu bemerken (siehe z.B. Reinecke 1961, Meixner 1982). Lunds Arbeit (Lund 2012) fasst die Kritik an dem Salonbegriff zwar zusammen, versucht allerdings auch mit diesem Begriff zu arbeiten. Dies geschieht nicht von ungefähr. Der ,jüdische Salon“ um 1800 ist inzwischen zu einem Berliner und Wiener Gedächtnisort geworden. Dafür zeugen nicht allein die aktuellen touristischen Hinweise in beiden Städten, sondern auch die vielfachen Versuche der letzten Jahre, den jüdischen Salon auch in einem neuen, diesmal nichtjüdischen Kontext in Berlin und Wien wiederzubeleben (vgl. z.B. Peham 2013; http://www.salonkultur.de/).

\section{Literaturverzeichnis}

Altenhofer, Norbert: „Geselligkeit als Utopie. Rahel und Schleiermacher“. Berlin zwischen 1789 und 1848: Facetten einer Epoche. Ausstellung der Akademie der Künste vom 30 August bis zum 1. November 1981. Berlin: Akademie der Künste, 1981, 37-42.

Arendt, Hannah: Elemente und Ursprünge totalitärer Herrschaft: Antisemitismus, Imperialismus, totale Herrschaft. München: Piper, 1955. 
Arendt, Hannah: Rahel Varnhagen. Lebensgeschichte einer deutschen Jüdin in der Romantik. Mit einer Auswahl von Rahel-Briefen und zeitgenössischen Abbildungen. München: Piper, 1959.

Arndt, Andreas: „Geselligkeit und Gesellschaft. Die Geburt der Dialektik aus dem Geist der Konversation in Schleiermachers ,Versuch einer Theorie des geselligen Betragens"“. Schultz, Hartwig (Hrsg.): Salons der Romantik. Beiträge eines Wiepersdorfer Kolloquiums zu Theorie und Geschichte des Salons. Berlin: De Gruyter, 1997, 45-61.

Bilski, Emily D.; Braun, Emily (Hrsg): Jewish Women and Their Salons: The Power of Conversation. New Haven: Yale University Press, 2005.

Bosold, Birgit (Hrsg.): Friederike Liman: Briefwechsel mit Rahel Levin Varnhagen und Karl Gustav von Brinckmann sowie Aufzeichnungen von Rahel Levin Varnhagen und Karl August Varnhagen. Eine historisch-kritische Edition mit Nachwort. http://www.sub.unihamburg.de/opus/volltexte/1996/395/ (04.11.2009).

Bruyn, Günter de (Hrsg.): Rahels erste Liebe. Rahel Levin und Karl Graf von Finckenstein in ihren Briefen. Berlin: Buchverlag Der Morgen, 1985.

Drewitz, Ingeborg: Berliner Salons: Gesellschaft und Literatur zwischen Aufklärung und Industriezeitalter. Berlin: Haude \& Spener, 1965.

Feilchenfeldt, Konrad: „,Berliner Salon` und Briefkultur um 1800“. Der Deutschunterricht 4 (1984), 77-99.

Feilchenfeldt, Konrad: „Rahel Varnhagens ,Geselligkeit‘ aus der Sicht Varnhagens. Mit einem Seitenblick auf Schleiermacher". Schultz, Hartwig (Hrsg.):Salons der Romantik. Beiträge eines Wiepersdorfer Kolloquiums zu Theorie und Geschichte des Salons. Berlin: De Gruyter, 1997, 147-169.

Frohberg, Regina: Schmerz der Liebe. Berlin: Salfeld, 1810.

Hahn, Barbara: Antworten Sie mir. Rahel Levin Varnhagens Briefwechsel. Basel: Stroemfeld-Roter Stern, 1990.

Hahn, Barbara: „Mit Goethe im Bad. Begegnungen im Exterritorialen: Rahel Levin Varnhagen, Sara und Marianne Meyer". Monatshefte 92,3 (2000), 336-350.

Hahn, Barbara: Die Jüdin Pallas Athene. Auch eine Theorie der Moderne. Berlin: Berlin Verlag, 2002. Hertz, Deborah: Jewish High Society in Old Regime Berlin. New Haven: Yale University Press, 1988. Dt. Übers.: Die jüdischen Salons im alten Berlin. Übersetzt von Gabriele Neumann-Kloth. Frankfurt a.M.: Hain, 1991.

Herz, Henriette: „Jugenderinnerungen von Henriette Herz“. Hrsg. von Heinrich Hahn. Mitteilungen aus dem Literaturarchiv Berlin 1 (1896), 141-184.

Herz, Henriette: Erinnerungen, Briefe und Zeugnisse. Neu hrsg. von Rainer Schmitz. Berlin: Die Andere Bibliothek, 2013.

Isselstein, Ursula: „Die Titel der Dinge sind das Fürchterlichste! Rahel Levins ,Erster Salon““. Schultz, Hartwig (Hrsg.):Salons der Romantik. Beiträge eines Wiepersdorfer Kolloquiums zu Theorie und Geschichte des Salons. Berlin: De Gruyter, 1997, 171-212.

Klein, Hans-Günter (Hrsg.): Die Musikveranstaltungen bei den Mendelssohns - Ein musikalischer Salon? Die Referate des Symposiums am 2. September 2006 in Leipzig. Leipzig: Mendelssohn Haus, 2007.

Klein, Hans-Günter: „,... als unsrer geistreichsten Landsleute einen“. Lea Mendelssohn Bartholdys Briefe an Carl Gustaf von Brinkman aus den Jahren 1811-1822“. Jahrbuch des Staatlichen Instituts für Musikfoschung Preußischer Kulturbesitz 2005, 243-266.

Lund, Hannah Lotte: Der Berliner „Jüdische Salon“ um 1800. Emanzipation in der Debatte. Berlin: De Gruyter, 2012.

Meixner, Horst: „Berliner Salons als Ort deutsch-jüdischer Symbiose“. Gegenseitige Einflüsse deutscher und jüischer Kultur von der Epoche der Aufklärung bis zur Weimarer Republik. Jahrbuch des Instituts für deutsche Geschichte, Beiheft 4. Tel Aviv: Univ. of Tel Aviv, 1982, 97-109. 
Mendelssohn Bartholdy, Lea: Ewig die Deine. Briefe an Henriette von Pereira-Arnstein. Hrsg. von Wolfgang Dinglinger und Rudolf Elvers. 2 Bde. Hannover: Wehrhahn, 2010.

Peham, Helga: Die Salonièren und die Salons in Wien: 200 Jahre Geschichte einer besonderen Institution. Graz: Styria Premium, 2013.

Reinecke, Hans: „Berliner Salons um 1800. Henriette Herz und Rahel Levin“. Der Monat 13, 151 (1961), 46-54.

Richarz, Monika: „In Familie, Handel und Salon: Jüdische Frauen vor und nach der Emanzipation der deutschen Juden“. Hausen, Karin; Wunder, Heide (Hrsg.): Frauengeschichte Geschlechtergeschichte. Frankfurt a.M., New York: Campus, 1992, 57-66.

Schadow, Johann Gottfried: Kunst-Werke und Kunst-Ansichten. Berlin: Decker, 1849.

Schleiermacher, Friedrich Daniel Ernst: „Versuch einer Theorie des geselligen Betragens“. Schleiermacher, Schriften aus der Berliner Zeit 1796-1799. Hrsg. von Günter Meckenstock. Krit. Gesamtausg. Abt. I/2. Berlin: De Gruyter, 1984, 163-184.

Schleiermacher, Friedrich Daniel Ernst: „Briefe bei Gelegenheit der politisch theologischen Aufgabe und des Sendschreibens jüdischer Hausväter“. Schleiermacher, Schriften aus der Berliner Zeit 1796-1799. Hrsg. von Günter Meckenstock. Krit. Gesamtausg. Abt. I/2. Berlin: De Gruyter, 1984, 329-413.

Schleiermacher, Friedrich Daniel Ernst: Briefwechsel und biographische Dokumente. Hrsg. von Andreas Arndt und Wolfgang Virmond. Krit. Gesamtausg. Abt. V/3. Berlin: De Gruyter, 1992.

Seibert, Peter: Der literarische Salon. Literatur und Geselligkeit zwischen Aufklärung und Vormärz. Stuttgart: Metzler, 1993.

Spiel, Hilde: Fanny Arnstein oder die Emanzipation. Frankfurt a.M.: S. Fischer, 1962.

Strube, Rolf (Hrsg.): Sie saßen und tranken am Teetisch. Anfänge und Blütezeit der Berliner Salons 1789-1871. München: Piper, 1992.

Varnhagen, Karl August: Denkwürdigkeiten I-III. Hrsg. von Konrad Feilchenfeldt. Frankfurt a.M.: Deutscher Klassiker Verlag, 1987.

Varnhagen, Rahel Levin: Briefe an eine Freundin. Rahel Varnhagen an Rebecca Friedländer. Hrsg. von Deborah Hertz. Köln: Kiepenheuer \& Witsch, 1989.

Varnhagen, Rahel Levin: Briefwechsel mit Pauline Wiesel. Hrsg. von Barbara Hahn. München: C.H. Beck, 1996.

Varnhagen, Rahel Levin: Briefwechsel mit Ludwig Robert. Hrsg. von Consolina Vigliero. München: C.H. Beck, 2001.

Varnhagen, Rahel Levin: Familienbriefe. Hrsg. von Renata Buzzo Márgari Barovero. München: C.H. Beck, 2009.

Varnhagen, Rahel [Levin]: Ein Buch des Andenkens für ihre Freunde. Hrsg. von Barbara Hahn nach einer Edition von Karl August Varnhagen. Mit einem Essay von Brigitte Kronauer. 6 Bde. Göttingen: Wallstein, 2011.

Weissberg, Liliane: „Kein Ort, nirgends: Gedanken zum jüdischen Salon“. Jahrbuch für Historische Bildungsforschung 9 (2003), 119-144.

Weissberg, Liliane: „Zur Pathologie des Salons. Regina Frohberg, Rahel Levin, Karl August Varnhagen und der Schmerz der Liebe“. Becker, Sabina (Hrsg.): Rahel Varnhagen. Studien zu ihrem Werk im zeitgenössischen Kontext. Saarbrücken: Röhrig Universitätsverlag, 2001, 119-161.

Wilhelmy, Petra: Der Berliner Salon im 19. Jahrhundert (1780-1914). Berlin: De Gruyter, 1989. Wilhelmy-Dollinger, Petra: Die Berliner Salons. Mit kulturhistorischen Spaziergängen. Berlin: De Gruyter, 2000.

Salonkultur. http://www.salonkultur.de/ (31.08.2015). 